\title{
Systematic review and meta-analysis on the incidence of delirium in intensive care unit inpatients after cognitive exercise intervention
}

\author{
Chaohong Xu, Zhenzhen Chen, Luxia Zhang, Han Guo
}

Intensive Medicine Department, The First People's Hospital of Wenling (The Affiliated Wenling Hospital of Wenzhou Medical University), Wenling, China

Contributions: (I) Conception and design: C Xu, H Guo; (II) Administrative support: Z Chen; (III) Provision of study materials or patients: C Xu, Z Chen, L Zhang; (IV) Collection and assembly of data: All authors; (V) Data analysis and interpretation: All authors; (VI) Manuscript writing: All authors; (VII) Final approval of manuscript: All authors.

Correspondence to: Han Guo. No. 333, Chuan'an South Road, Chengxi Street, Wenling, China. Email: xueyi8416@163.com.

Background: The incidence of delirium in patients in the intensive care unit (ICU) is relatively high. Current research results on the effect of cognitive exercise on the incidence of delirium in ICU inpatients are inconsistent. In this study, a meta-analysis was performed on the impact of cognitive exercise on the incidence of delirium in ICU inpatients to provide an evidence-based reference for the clinical prevention of delirium.

Methods: Articles were searched in PubMed, MEDLINE, the Cochrane Library, Chinese National Knowledge Infrastructure (CNKI), China Biology Medicine Disc (CBMD), Wanfang Database, and Western Biomedical Journal Database. The search strategy and search terms for Chinese and English databases were as follows: cognitive exercises or activity or functional exercise or rehabilitation or active intervention or gradual training or physical therapy or physical therapy, delirium, and randomized controlled trial (RCT) or randomization. Two researchers were required to independently screen the articles, extract data, and repeatedly assess the risk of bias for the included articles.

Results: A total of 7 studies were included. The incidence of delirium was tested for heterogeneity, which showed that $\mathrm{I}^{2}=94 \%>50 \%$ and $\mathrm{P}<0.00001$, indicating heterogeneity among studies. There was no significant difference between the treatment group and the routine group $[Z=1.28$, odds ratio $(O R)=0.43$, $95 \%$ confidential interval (CI): $0.12-1.58, \mathrm{P}=0.20]$. The duration of delirium in treatment group and routine group was significantly different $[\mathrm{Z}=3.24$, mean difference $(\mathrm{MD})=-1.99,95 \% \mathrm{CI}:-3.20,-0.79, \mathrm{P}=0.001]$. The heterogeneity test was conducted for the length of hospitalization, showing that Chi-squared test $\left(\mathrm{Chi}^{2}\right)=2.16$, degree of freedom $(\mathrm{df})=4, \mathrm{I}^{2}=0 \%<50 \%$, and $\mathrm{P}=0.71$, indicating that the heterogeneity of each study group was acceptable. The difference between the treatment group and the routine group was statistically significant $(\mathrm{Z}=10.84, \mathrm{MD}=-2.10,95 \% \mathrm{CI}:-2.48,-1.72, \mathrm{P}<0.00001)$.

Discussion: Meta-analysis results confirmed that cognitive exercises can reduce the incidence and duration of delirium in ICU inpatients, and shorten the length of hospitalization.

Keywords: Cognitive exercises; intensive care unit; delirium; incidence; meta-analysis

Submitted Dec 11, 2021. Accepted for publication Feb 16, 2022.

doi: 10.21037/apm-21-3938

View this article at: https://dx.doi.org/10.21037/apm-21-3938 


\section{Introduction}

Delirium is a fast-onset brain syndrome with non-specific etiology. Its main manifestations are disturbances in consciousness, attention, perception, memory, thinking, mood, sleep cycles, and abnormal psychomotor behaviors, with uncertain duration and severity (1). Intensive care unit (ICU) delirium is a common clinical syndrome in the treatment of critically ill hospitalized patients. ICU patients in the special environment of disease and intensive care unit may be the side effects of drugs, or they need to face great pressure and fear every day, which leads to delusions, hallucinations, delusions, eccentric behaviors, excessive vigilance and even delirium (2-4). In the clinical environment, the incidence of delirium is $10-60 \%$ and the incidence in ICU inpatients is even as high as $60-82 \%$, while the incidence of mechanically ventilated patients is the highest (5). Delirium can increase the mortality, length of hospitalization, and medical expenses of critically ill patients. After being discharged from hospital, patients with delirium during their stay in the ICU have a significantly lower quality of life (6-8). Delirium is one of the risk factors for cognitive impairment in ICU inpatients. Patients with delirium are more likely to suffer from long-term cognitive dysfunction in the ICU, which severely hinders the patient's recovery progress and leads to an increase in the readmission rate (9).

Currently, there is no uniform plan for the treatment of delirium. The efficacy of medication in the treatment of delirium is not obvious, the cost of medication is expensive, and it shows inconsistent efficacy compared to other nondrug treatments (10). During the bed rest period of patients, activity decreases, protein synthesis slows down, and oxidative stress is promoted, which will increase the activity of proteolytic enzymes, accelerate protein degradation, accelerate muscle dysfunction, and increase the incidence of delirium in the ICU (11). Through cognitive function exercise, directional training of patients' psychology and behavior, and evaluation by Wechsler Adult Intelligence Scale and Wechsler Adult Memory Scale can improve patients' real perception and directional ability of things around them and reduce patients' fear of unfamiliar environment. In addition, cognitive exercises can reduce the protein degradation caused by oxidative stress in patients, thereby preventing the occurrence of delirium in the ICU (12). The specific content of cognitive exercise evaluation includes initial evaluation and continuous evaluation, and its implementation plans include progressive functional exercises and goal-oriented functional exercises. Studies have shown that cognitive exercises can reduce the mechanical ventilation time and length of hospitalization of patients, and improve their muscle strength and ability of daily living. The guidelines for sedation, analgesia, and delirium suggest that cognitive exercises in ICU inpatients can effectively promote the recovery of patients. Therefore, it is necessary for ICU inpatients to perform cognitive exercises.

At present, there has been a systematic evaluation on the influence of cognitive function exercise on delirium of ICU patients, but there are many intervention measures, and the quality of literature is uneven; In addition, the conclusions of the study are not uniform. The innovation of this study lies in the use of Cochrane system to comprehensively search and collect the randomized controlled trials (RCTs) of delirium in ICU patients after intervention of cognitive function exercise for systematic evaluation and metaanalysis, in order to provide evidence-based medical basis for prevention and management of delirium in ICU.

We present the following article in accordance with the PRISMA reporting checklist (available at https://apm. amegroups.com/article/view/10.21037/apm-21-3938/rc).

\section{Methods}

\section{Article retrieval}

English articles were searched in PubMed, MEDLINE, and the Cochrane Library, and Chinese articles were searched in Chinese National Knowledge Infrastructure (CNKI), China Biology Medicine Disc (CBM), Wanfang Database, and Western Biomedical Journal Database. The search strategy and search terms for Chinese and English databases were as follows: cognitive exercises or activity or functional exercise or rehabilitation or active intervention or gradual training or physical therapy or physical therapy, delirium, and RCT or randomization.

Subject words and free words were combined, aiming to include more relevant articles. RCTs on the occurrence of delirium in ICU inpatients after the intervention of cognitive exercises published from the establishment of the database to June 28, 2021 were retrieved.

\section{Inclusion and exclusion criteria of the included articles}

The inclusion criteria of articles were as follows: articles which were prospective RCTs, without restrictions on allocation concealment and blinding; articles with adult patients with delirium in the ICU as the research subjects; 
articles with intervention of cognitive exercises on the basis of routine care, including active or passive exercise on the bed, sitting on the bed, standing on the bed, sitting on the bedside chair, and assisted walking in the experimental group, and giving routine care, including basic care, health education, specialist care, and intensive care in the routine group; and articles which mentioned the outcome indicators of the incidence of delirium, duration of delirium, deliriumfree time, and hospitalization days. Medical staff in the ICU evaluated patients' consciousness, underlying diseases, respiratory status, muscle strength, activity intensity, pain, and developed cognitive exercise plans, including passive exercises and active exercises.

Articles meeting the following criteria were excluded: articles which were non-prospective RCTs; articles with research subjects who were not ICU inpatients; articles with the intervention measures of no cognitive exercises combined with other methods; articles which were published repeatedly; and articles whose authors could not be contacted to obtain relevant outcome data.

\section{Outcome indicators}

The outcome indicators included the incidence of delirium, duration of delirium, delirium-free time, and hospitalization days.

\section{Article screening and data extraction}

Two professionals were required to use a unified Microsoft Excel table to independently filter articles, read the titles and abstracts of articles, extract data, and then cross-check them. If they encountered differences, it would be resolved through discussion. The main data extracted included: general information of the articles (journal, publication data, title, first author, and country), basic information of the research subjects (gender, age, and sample size), intervention measures (specific operation and follow-up time), major factors for the risk of bias, and extraction of outcome indicators (incidence of delirium, duration of delirium, delirium-free time, and hospitalization days).

\section{Evaluation of the risk of bias}

Two researchers were required to evaluate the methodological quality of the included articles using the bias risk assessment tool provided by the Cochrane Handbook Version 5.1.0 (13). If there was a disagreement, a consensus conclusion would be obtained through discussion. Evaluation items included determination of the random sequence generation method; allocation concealment; researcher blinding and research outcome blinding; loss to follow-up and withdrawal; judgment selection bias; and other biases. Each item was evaluated as "low risk of bias", "unclear risk of bias", and "high risk of bias". The included articles which fully met the above criteria were defined as grade A, suggesting that the possibility of various biases was low. Articles satisfying the above quality standards were defined as grade $\mathrm{B}$, indicating that the possibility of bias was moderate. Original articles which did not meet the above quality standards at all were defined as grade $\mathrm{C}$, indicating that the possibility of bias was high.

\section{Sensitivity analysis}

We also aimed to study the impact of each study on the total combined effect size, and excluded the more influential studies. The random effect model (REM) and fixed effect model (FEM) were used to compare the results. According to the consistency of the results, the reliability of the combined results was analyzed and the funnel plot was used to determine whether there was publication bias.

\section{Statistical analysis}

The risk of bias evaluation chart of RevMan 5.3 software provided by the Cochrane Collaboration was used to evaluate the risk of bias of the included articles, and the data was sorted, filtered, and then input into the software to draw the plot. Binary variables were analyzed using odds ratio (OR) and $95 \%$ confidential interval (CI) as effect indicators, and continuous variables were analyzed by taking the mean difference (MD), standardized MD (SMD), and 95\% CI as the effect indicators. The heterogeneity among the articles was explored using the $\mathrm{I}^{2}$ test. When $\mathrm{P}>0.01$ and $\mathrm{I}^{2} \leq 50 \%$, it indicated that the heterogeneity of the included articles was small, and the FEM was used for meta-analysis. When $\mathrm{P}<0.01$ and $\mathrm{I}^{2}>50 \%$, it indicated that the included articles showed obvious heterogeneity, and the REM was used for meta-analysis.

\section{Results}

Search results and basic information of the included articles

A total of 459 articles were obtained by searching the 


\section{Identification of studies via databases and registers}

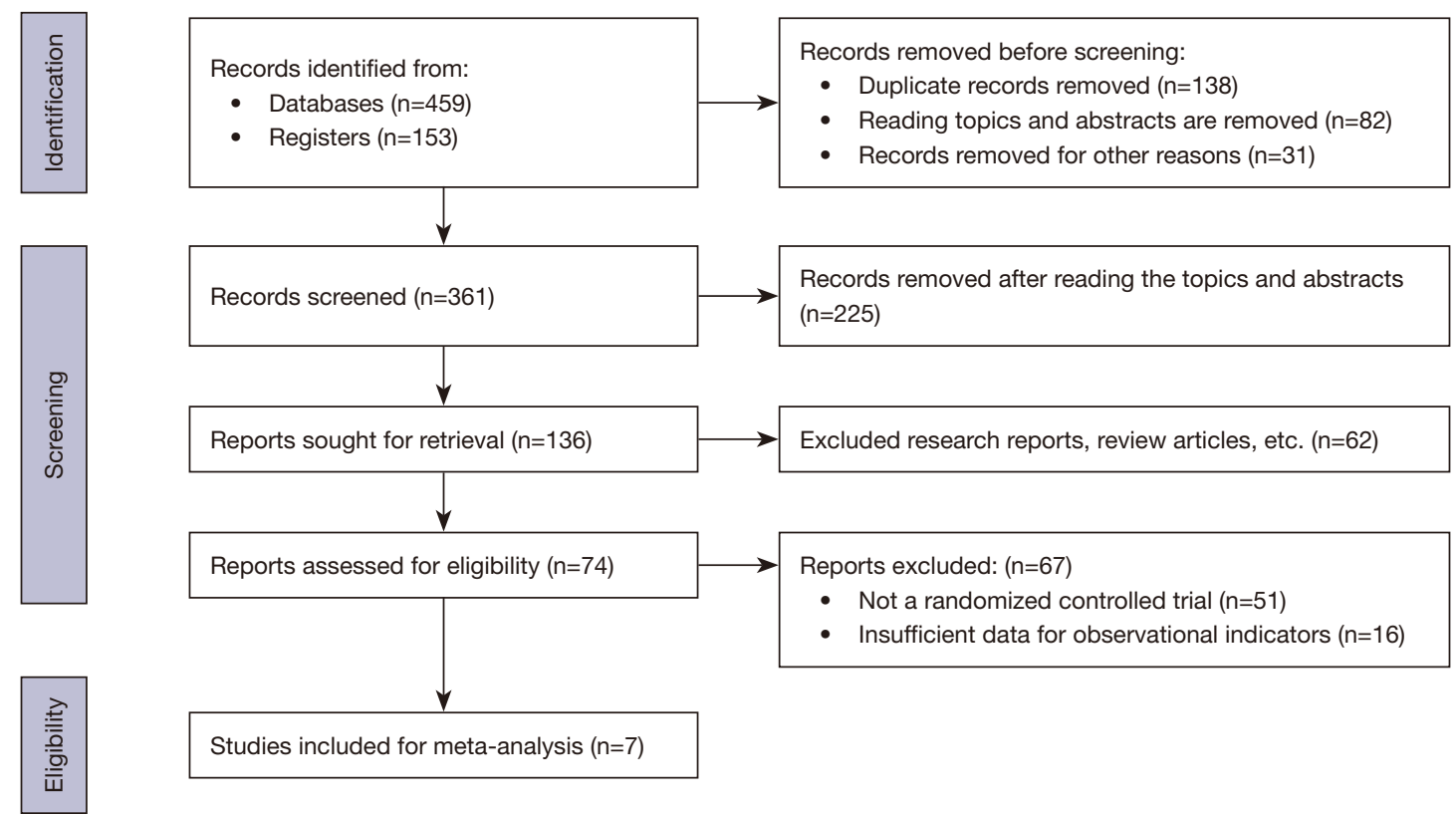

Figure 1 Flowchart of the article retrieval process.

databases and 153 articles were obtained by registers. Of these, 138 articles that were duplicate articles were eliminated, 82 articles were not qualified after the titles and abstracts were read, and 31 articles were eliminated for other reasons. After the selection of titles and abstracts, 361 papers were included. After the full texts were read, 225 articles were removed, leaving 136 articles. There were 62 research reports and reviews, and 74 papers remained. A total of 51 non-RCTs articles were excluded, and 16 articles were excluded because the relevant information of the research could not be further extracted. Seven articles were finally included in the meta-analysis. Figure 1 shows the flowchart of the article retrieval process.

There were 7 articles that met the inclusion criteria, involving 1,291 patients. Among the 7 articles, the sample size ranged from 94 to 300 . The incidence of delirium, duration of delirium, delirium-free time and hospitalization days were described in detail in the 7 articles. Table 1 shows the basic characteristics and scale scores of the included articles.

\section{Risk of bias evaluation results of the included articles}

Figure 2 shows the risk of bias evaluation results of the included articles drawn by Rev Man 5.3 software. Figure 3 is a summary diagram of the risk of bias of the articles. Among the 7 RCTs in this study, 1 RCT described allocation concealment in detail, 7 described the correct random allocation method, and 1 article did not use blinding. The quality evaluation results of all articles were grade B.

\section{Meta-analysis on the incidence of delirium}

A total of 4 articles (16,18-20), analyzed the incidence of delirium in the RCTs and their relative risk. The 4 articles described the incidence of delirium using dichotomous variables. Figure 4 is a forest plot for incidence of delirium using REM. The overall heterogeneity test was performed, and the results revealed $\mathrm{I}^{2}=94 \%>50 \%$ and $\mathrm{P}<0.00001$, so the heterogeneity among the articles was acceptable. The REM analysis results showed that the treatment group and the routine group were not significantly different in terms of the incidence of delirium $(\mathrm{Z}=1.28, \mathrm{OR}=0.43,95 \% \mathrm{CI}$ : $0.12-1.58, \mathrm{P}=0.20)$. These findings showed that cognitive exercises could reduce the incidence of delirium in the ICU.

Figure 5 is a funnel plot of the incidence of delirium. The distribution of most articles was relatively concentrated, with only a few scattered, indicating that there was no 
Table 1 Basic characteristics and scale scores of the included articles

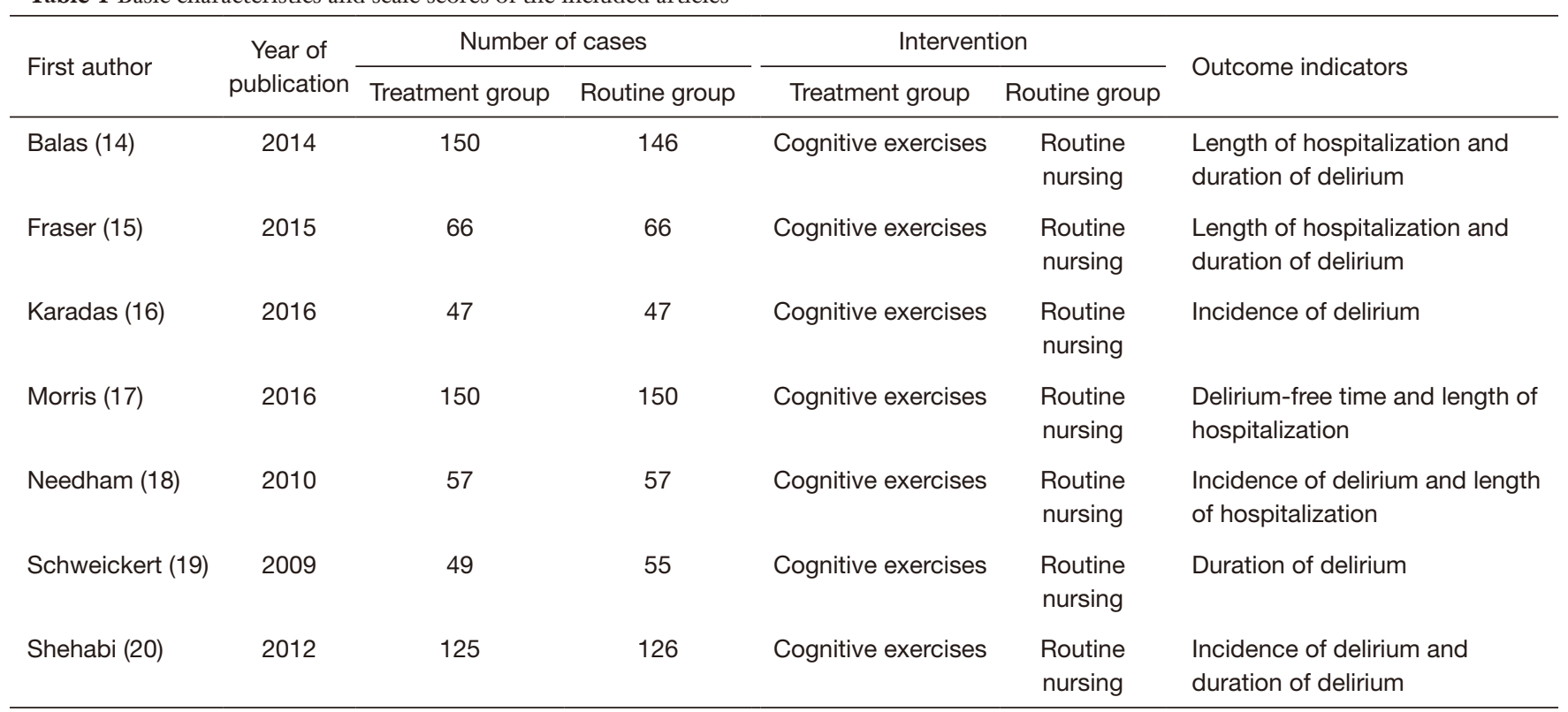

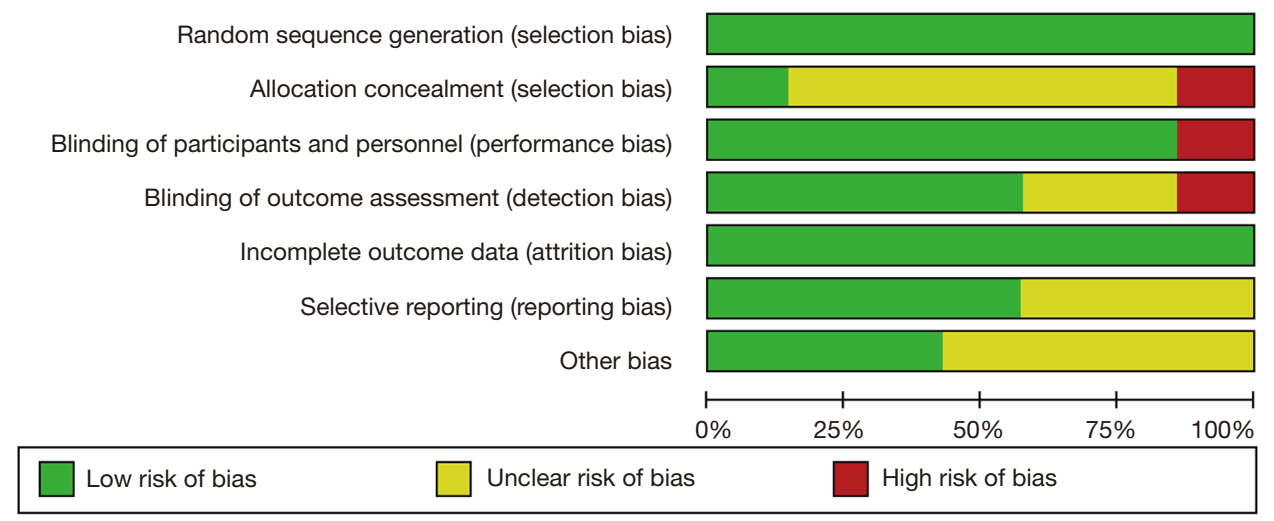

Figure 2 Risk of bias evaluation results of the included articles.

publication bias in the articles included in this study.

\section{Meta-analysis on the duration of delirium}

A total of 3 articles $(14,19,20)$ analyzed the duration of delirium in the RCTs and their relative risk. Figure 6 is a forest plot for the duration of delirium using REM. The overall heterogeneity test was performed, and the results revealed $\mathrm{Chi}^{2}=80.08, \mathrm{df}=2, \mathrm{I}^{2}=98 \%$ and $\mathrm{P}<0.00001$, so the heterogeneity among the articles was acceptable. The REM analysis results showed that the treatment group and the routine group were significantly different in terms of the incidence of delirium $(\mathrm{Z}=3.24, \mathrm{MD}=-1.99,95 \% \mathrm{CI}:-3.20$, $-0.79, \mathrm{P}=0.001)$.

Figure 7 is a funnel plot of the duration of delirium. The distribution of most articles was relatively concentrated, with only a few scattered, indicating that there was no publication bias in the articles included in this study.

\section{Meta-analysis on the delirium-free time}

A total of 2 articles $(15,17)$ analyzed the delirium-free time in the RCTs and their relative risk. Figure 8 is a forest plot for the delirium-free time using REM. The 
overall heterogeneity test was performed, and the results revealed $\mathrm{Chi}^{2}=11.06, \mathrm{df}=1, \mathrm{I}^{2}=91 \%$ and $\mathrm{P}=0.0009$, so the heterogeneity among the articles was acceptable. The REM analysis results showed that the treatment group and the routine group were no significantly different in terms of the delirium-free time $(\mathrm{Z}=0.97, \mathrm{MD}=-0.11,95 \% \mathrm{CI}:-0.34$, $0.12, \mathrm{P}=0.33)$.

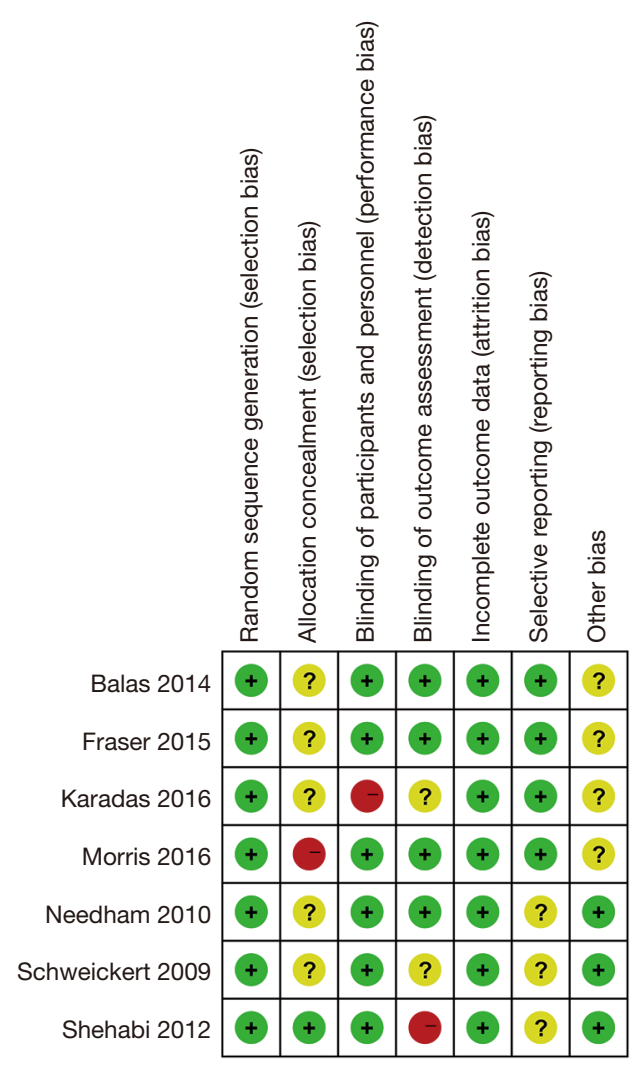

Figure 3 Summary of the risk of bias evaluation of the articles. +, low risk; -, high risk; ?, no clear.
Figure 9 is a funnel plot of the duration of delirium. The distribution of most articles was relatively concentrated, with only a few scattered, indicating that there was no publication bias in the articles included in this study.

\section{Meta-analysis on the length of hospitalization}

A total of 5 articles $(14,15,17-19)$ analyzed the length of hospitalization and MD of RCTs. Figure 10 shows the forest plot of the length of hospitalization using FEM. Five articles used continuous variables to describe the length of hospitalization. There were 946 cases in total, including 472 cases in the treatment group and 474 cases in the routine group. The overall heterogeneity test was performed, and it was found that $\mathrm{Chi}^{2}=2.16, \mathrm{df}=4, \mathrm{I}^{2}=0 \%<50 \%$, and $\mathrm{P}=0.71$, so the heterogeneity was acceptable. The FEM analysis results demonstrated that the difference between the 2 groups was statistically significant $(\mathrm{Z}=10.84, \mathrm{MD}=-2.10,95 \% \mathrm{CI}:-2.48$, $-1.72, \mathrm{P}<0.00001)$. This indicates that cognitive exercises can significantly shorten the length of hospitalization of patients with delirium in the ICU. The 2 included articles were eliminated one by one and then subjected to sensitivity analysis. The results showed that the combined effect size did not change significantly, indicating that the analysis results of the outcome index were very stable and were not affected by a single study.

Figure 11 is the funnel plot of the length of hospitalization. The distribution of most articles was relatively concentrated and basically symmetrical, indicating that there was no publication bias in the articles included in this study.

\section{Discussion}

A total of 7 RCTs on cognitive exercise intervention for the treatment of delirium in the ICU were included in this

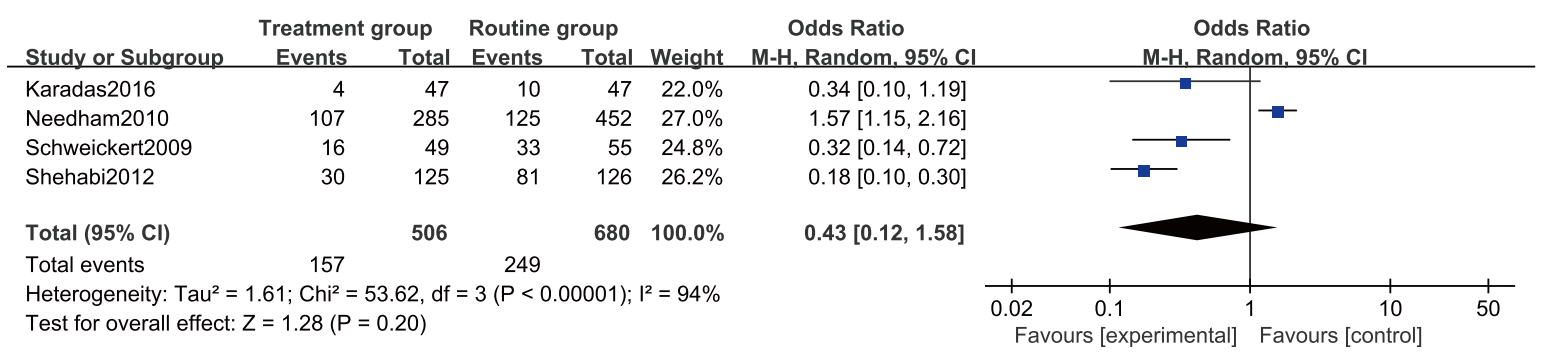

Figure 4 Forest plot for the incidence of delirium using REM. REM, random effect model; CI, confidence interval; Chi ${ }^{2}$, chi-square test; df, degree of freedom. 


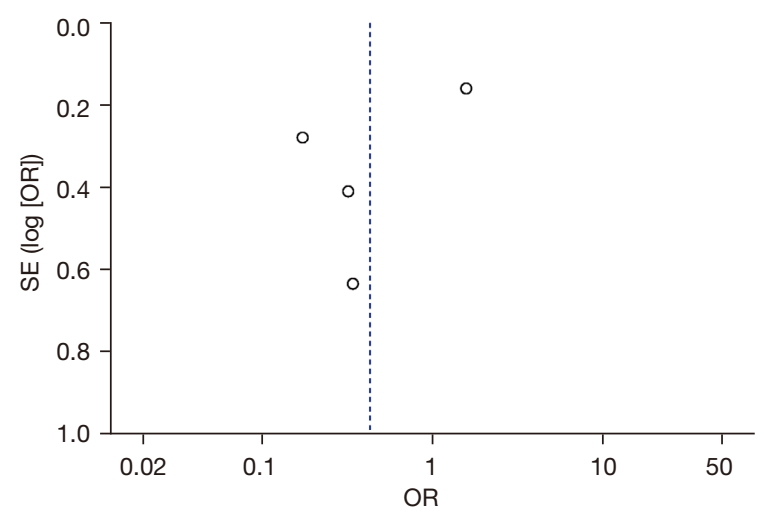

Figure 5 Funnel plot of the incidence of delirium. SE, standard error; OR, odds ratio.

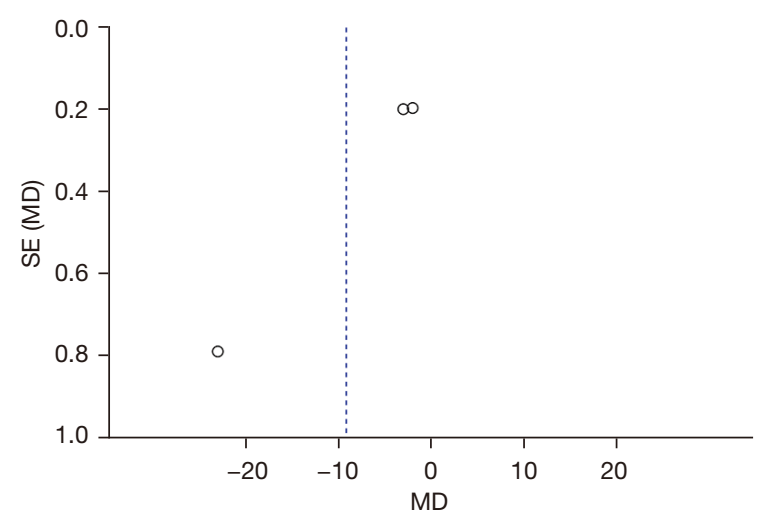

Figure 7 Funnel plot of the duration of delirium. SE, standard error; MD, mean difference.

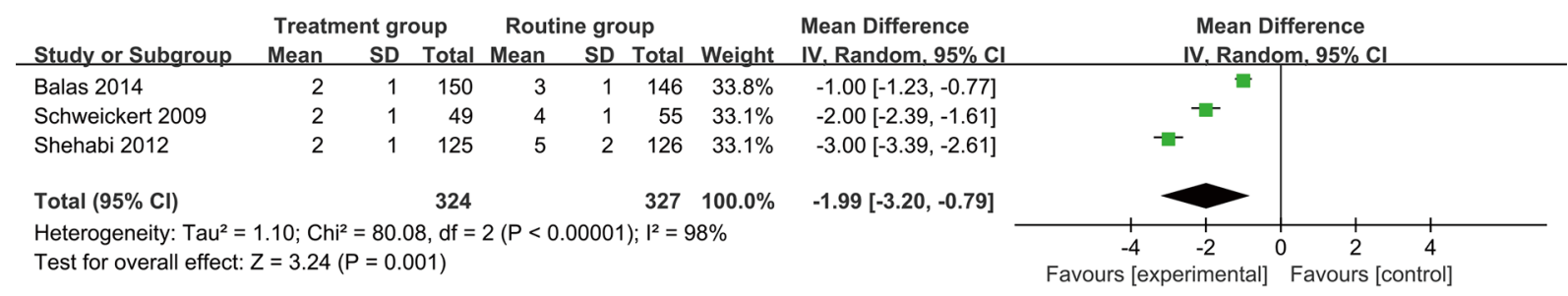

Figure 6 Forest plot for the duration of delirium using REM. REM, random effect model; CI, confidence interval; Chi ${ }^{2}$, chi-square test; df, degree of freedom; SD, standard deviation.

study, of which 1 RCT described allocation concealment in detail, 7 described the correct random allocation method, and 1 article did not use blinding. The results showed that there was no significant difference between the baseline data of the treatment group and the routine group $(\mathrm{P}>0.05)$, indicating that they were comparable.

The incidence and ORs of delirium in the 4 RCTs were analyzed. Meta-analysis results showed that cognitive exercise significantly reduced the incidence of delirium in ICU inpatients $(Z=1.28, O R=0.43,95 \%$ CI: $0.12-1.58$, $\mathrm{P}=0.20)$, which was consistent with Thomason et al. [2004] (21). Cognitive exercise in ICU patients can improve their mobility, enhance their self-confidence, improve their psychological status, and enhance their belief in recovery. Based on the existing research findings, clinical experience, and expert consensus, cognitive exercise can significantly reduce patients' medical costs (22-25). A total of 2 studies analyzed the length of stay and MD. When delirium occurs, it can have serious short-term and long-term effects on ICU patients, such as increased mortality, increased medical costs, longer hospital stays, cognitive impairment, and post-traumatic stress reactions (26-28). Tatematsu et al.'s [2011] (29) research pointed out that cognitive function exercise combined with drug treatment for delirium was better than cognitive function exercise alone. Meta-analysis showed that cognitive exercise significantly shortened the length of hospital stay in ICU patients with delirium $(\mathrm{Z}=10.84, \mathrm{MD}=-2.10,95 \% \mathrm{CI}:-2.48,-1.72, \mathrm{P}<0.00001)$. Cognitive exercise can promote the recovery of patients, enable them to return to society as soon as possible, and effectively improve the quality of life of patients in the later stage, which is consistent with the analysis results of Rosa et al. [2018] (30). After the patients were discharged from hospital, the longitudinal study was conducted by extending the follow-up time, and it was found that the long-term effect of cognitive function exercise was better than that of routine nursing.

After the included articles were eliminated one by one, sensitivity analysis was performed, and the results showed that the combined effect size did not change significantly, 


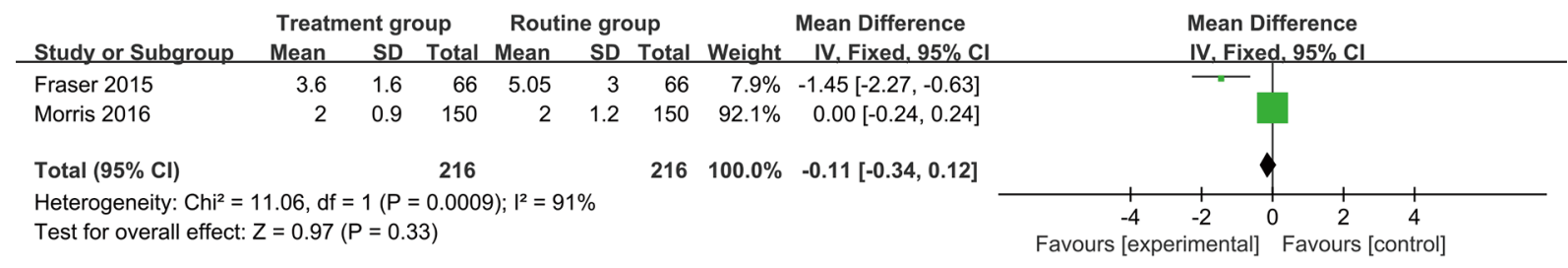

Figure 8 Forest plot for the delirium-free time using REM. REM, random effect model; CI, confidence interval; Chi ${ }^{2}$, chi-square test; df, degree of freedom; SD, standard deviation.

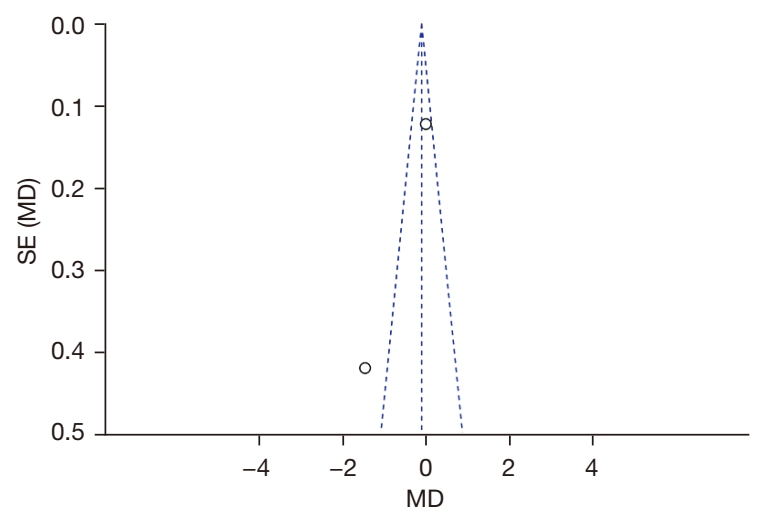

Figure 9 Funnel plot of the delirium-free time. SE, standard error; MD, mean difference.



Figure 11 Funnel plot of the length of hospitalization. SE, standard error; MD, mean difference.

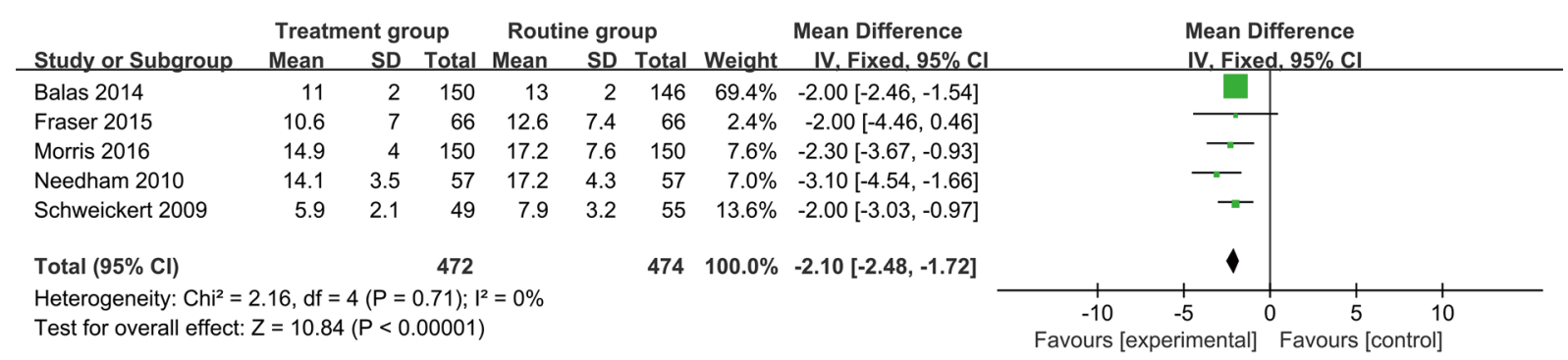

Figure 10 Forest plot for the length of hospitalization using FEM. FEM, fixed effect model; CI, confidence interval; Chi ${ }^{2}$, chi-square test; df, degree of freedom; SD, standard deviation.

indicating that the analysis results of the outcome indicators were very stable and were not affected by a single study. The circles were scattered around the midline and were roughly symmetrical, and there was no bias in the publications. Generally speaking, cognitive exercises in ICU inpatients, whether active or passive exercises, were beneficial for reducing the incidence of delirium and length of hospitalization. It not only saves medical resources, but also improves the outcome of patients. It is recommended to be widely used in clinical practice. However, cognitive exercise in ICU inpatients can be greatly affected by the sedative effect of drugs, and it is necessary to improve patient sedation management in the later stage to increase the possibility of exercise.

\section{Conclusions}

In this study, the relevant RCTs on cognitive exercise in 
ICU inpatients with delirium were screened for systematic evaluation and meta-analysis, aiming to explore the impact of cognitive exercises and routine nursing on the incidence of delirium. Meta-analysis results confirmed that cognitive exercises can reduce the incidence of delirium and duration of delirium in patients in the ICU, and shorten the length of hospitalization of patients. The limitation of this study was that there were few related studies on ICU inpatients after discharge, and follow-up visits need to be added to future studies to understand the long-term therapeutic effect of cognitive exercises on delirium. In conclusion, this study provides an evidence-based reference for the application of cognitive exercises in ICU inpatients.

\section{Acknowledgments}

Funding: None.

\section{Footnote}

Reporting Checklist: The authors have completed the PRISMA reporting checklist. Available at https://apm. amegroups.com/article/view/10.21037/apm-21-3938/rc

Conflicts of Interest: All authors have completed the ICMJE uniform disclosure form (available at https://apm. amegroups.com/article/view/10.21037/apm-21-3938/coif). The authors have no conflicts of interest to declare.

Ethical Statement: The authors are accountable for all aspects of the work in ensuring that questions related to the accuracy or integrity of any part of the work are appropriately investigated and resolved.

Open Access Statement: This is an Open Access article distributed in accordance with the Creative Commons Attribution-NonCommercial-NoDerivs 4.0 International License (CC BY-NC-ND 4.0), which permits the noncommercial replication and distribution of the article with the strict proviso that no changes or edits are made and the original work is properly cited (including links to both the formal publication through the relevant DOI and the license). See: https://creativecommons.org/licenses/by-nc-nd/4.0/.

\section{References}

1. Wassenaar A, Schoonhoven L, Devlin JW, et al. Delirium prediction in the intensive care unit: comparison of two delirium prediction models. Crit Care 2018;22:114.

2. Luetz A, Grunow JJ, Mörgeli R, et al. Innovative ICU Solutions to Prevent and Reduce Delirium and PostIntensive Care Unit Syndrome. Semin Respir Crit Care Med 2019;40:673-86.

3. Hayhurst CJ, Pandharipande PP, Hughes CG. Intensive Care Unit Delirium: A Review of Diagnosis, Prevention, and Treatment. Anesthesiology 2016;125:1229-41.

4. Denehy L, Skinner EH, Edbrooke L, et al. Exercise rehabilitation for patients with critical illness: a randomized controlled trial with 12 months of follow-up. Crit Care 2013;17:R156.

5. Álvarez EA, Garrido MA, Tobar EA, et al. Occupational therapy for delirium management in elderly patients without mechanical ventilation in an intensive care unit: A pilot randomized clinical trial. J Crit Care 2017;37:85-90.

6. Ely EW, Inouye SK, Bernard GR, et al. Delirium in mechanically ventilated patients: validity and reliability of the confusion assessment method for the intensive care unit (CAM-ICU). JAMA 2001;286:2703-10.

7. Smith HA, Brink E, Fuchs DC, et al. Pediatric delirium: monitoring and management in the pediatric intensive care unit. Pediatr Clin North Am 2013;60:741-60.

8. Hughes CG, Hayhurst CJ, Pandharipande PP, et al. Association of Delirium during Critical Illness With Mortality: Multicenter Prospective Cohort Study. Anesth Analg 2021;133:1152-61.

9. Pavone KJ, Jablonski J, Junker P, et al. Evaluating delirium outcomes among older adults in the surgical intensive care unit. Heart Lung 2020;49:578-84.

10. Marra A, Pandharipande PP, Patel MB. Intensive Care Unit Delirium and Intensive Care Unit-Related Posttraumatic Stress Disorder. Surg Clin North Am 2017;97:1215-35.

11. Weinhouse GL. Delirium and sleep disturbances in the intensive care unit: can we do better? Curr Opin Anaesthesiol 2014;27:403-8.

12. Gunther ML, Morandi A, Ely EW. Pathophysiology of delirium in the intensive care unit. Crit Care Clin 2008;24:45-65, viii.

13. Easton C, MacKenzie F. Sensory-perceptual alterations: delirium in the intensive care unit. Heart Lung 1988;17:229-37.

14. Balas MC, Vasilevskis EE, Olsen KM, et al. Effectiveness and safety of the awakening and breathing coordination, delirium monitoring/management, and early exercise/ mobility bundle. Crit Care Med 2014;42:1024-36.

15. Fraser D, Spiva L, Forman W, et al. Original Research: 
Implementation of an Early Mobility Program in an ICU. Am J Nurs 2015;115:49-58.

16. Karadas C, Ozdemir L. The effect of range of motion exercises on delirium prevention among patients aged 65 and over in intensive care units. Geriatr Nurs 2016;37:180-5.

17. Morris PE, Berry MJ, Files DC, et al. Standardized Rehabilitation and Hospital Length of Stay Among Patients With Acute Respiratory Failure: A Randomized Clinical Trial. JAMA 2016;315:2694-702.

18. Needham DM, Korupolu R, Zanni JM, et al. Early physical medicine and rehabilitation for patients with acute respiratory failure: a quality improvement project. Arch Phys Med Rehabil 2010;91:536-42.

19. Schweickert WD, Pohlman MC, Pohlman AS, et al. Early physical and occupational therapy in mechanically ventilated, critically ill patients: a randomised controlled trial. Lancet 2009;373:1874-82.

20. Shehabi Y, Bellomo R, Reade MC, et al. Early intensive care sedation predicts long-term mortality in ventilated critically ill patients. Am J Respir Crit Care Med 2012;186:724-31.

21. Thomason JW, Ely EW. Delirium in the intensive care unit is bad: what is the confusion? Crit Care Med 2004;32:2352-4.

22. Nelson S, Muzyk AJ, Bucklin MH, et al. Defining the Role of Dexmedetomidine in the Prevention of Delirium in the Intensive Care Unit. Biomed Res Int 2015;2015:635737.

23. Vasilevskis EE, Chandrasekhar R, Holtze CH, et al. The

Cite this article as: $\mathrm{Xu} \mathrm{C}$, Chen $\mathrm{Z}$, Zhang L, Guo H. Systematic review and meta-analysis on the incidence of delirium in intensive care unit inpatients after cognitive exercise intervention. Ann Palliat Med 2022;11(2):663-672. doi: 10.21037/ apm-21-3938
Cost of ICU Delirium and Coma in the Intensive Care Unit Patient. Med Care 2018;56:890-7.

24. Baumgartner L, Lam K, Lai J, et al. Effectiveness of Melatonin for the Prevention of Intensive Care Unit Delirium. Pharmacotherapy 2019;39:280-7.

25. Hermes C, Acevedo-Nuevo M, Berry A, et al. Gaps in pain, agitation and delirium management in intensive care: Outputs from a nurse workshop. Intensive Crit Care Nurs 2018;48:52-60.

26. Johnson K, Fleury J, McClain D. Music intervention to prevent delirium among older patients admitted to a trauma intensive care unit and a trauma orthopaedic unit. Intensive Crit Care Nurs 2018;47:7-14.

27. Brown KN, Soo A, Faris P, et al. Association between delirium in the intensive care unit and subsequent neuropsychiatric disorders. Crit Care 2020;24:476.

28. Ely EW, Margolin R, Francis J, et al. Evaluation of delirium in critically ill patients: validation of the Confusion Assessment Method for the Intensive Care Unit (CAM-ICU). Crit Care Med 2001;29:1370-9.

29. Tatematsu N, Hayashi A, Narita K, et al. The effects of exercise therapy on delirium in cancer patients: a retrospective study. Support Care Cancer 2011;19:765-70.

30. Rosa RG, Falavigna M, Robinson CC, et al. Study protocol to assess the effectiveness and safety of a flexible family visitation model for delirium prevention in adult intensive care units: a cluster-randomised, crossover trial (The ICU Visits Study). BMJ Open 2018;8:e21193.

(English Language Editor: C. Betlazar-Maseh) 